\title{
Free-Floating HI Clouds in the M 81 Group
}

\author{
Elias Brinks ${ }^{1}$, Fabian Walter ${ }^{2}$ and Evan D. Skillman ${ }^{3}$ \\ ${ }^{1}$ Centre for Astrophysics Research, University of Hertfordshire, College Lane, \\ Hatfield AL10 9AB, United Kingdom \\ email: E.Brinks@herts.ac.uk \\ ${ }^{2}$ Max-Planck-Institut für Astronomie, Königstuhl 17, 69117 Heidelberg, Germany \\ email: walter@mpia.de \\ ${ }^{3}$ Dept. of Astronomy, Univ. of Minnesota, 116 Church St. SE, Minneapolis, MN 55455, USA \\ email: skillman@astro.umn.edu
}

\begin{abstract}
Recent VLA observations pointed at dwarf spheroidal (dSph) galaxies in the M 81 group reveal a hitherto hidden population of extremely low mass $\left(\sim 10^{5} \mathrm{M}_{\odot}\right)$ HI clouds with no obvious optical counterparts. We have searched 10 fields in the M81 group totalling 2.2 square degree, both targeting known dwarf spheroidal galaxies and blank fields around the central triplet. Our observations show that the new population of low-mass HI clouds appears to be confined to a region toward the south-east of the central triplet (at distances of $\sim 100 \mathrm{kpc}$ from M 81). Possible explanations for these free-floating HI clouds are that they are related to the dSphs found to the South-East of M 81, that they belong to the galaxies of the M 81 triplet (equivalent to $\mathrm{HVCs}$ ), that they are of primordial nature and provide fresh, unenriched material falling into the M 81 group, or that they are tidal debris from the 3-body interaction involving M 81-M 82-NGC 3077. Based on circumstantial evidence, we currently favour the latter explanation.
\end{abstract}

Keywords. galaxies: individual (M81), galaxies: interactions, intergalactic medium, radio lines: galaxies

\section{Introduction}

The nearby M 81 group has been the subject of several surveys in the $21-\mathrm{cm}$ line of atomic hydrogen (HI). These surveys can be divided into two broad categories: targeted and blind. Appleton et al. (1981) using the 76-m Lowell telescope and later Yun et al. (1994; see also Yun et al. 2000) mapped the large-scale HI distribution in the group, tracing the complex tidal tails and bridges resulting from the 3-body interaction between M 81, M 82, and NGC 3077. Other targeted surveys, extending beyond the area covered by the triplet, have been those by Huchtmeier \& Skillman (1998) and Huchtmeier et al. (2000) to determine the HI content of optically selected objects such as dwarf galaxies. Motivations for blind surveys have varied from i) searches for High Velocity Clouds (HVC) and Compact HVC analogues and ii) deep surveys to push down the HI luminosity function, to iii) searching for "Dark Galaxies", i.e., dark matter haloes which have not as yet turned (part of) their gas content into stars. The first blind survey which included the M 81 group was that of Lo \& Sargent (1979). Recently, Boyce et al. (2001) have repeated this at improved resolution and sensitivity as part of HIJASS, the HI Jodrell All-Sky Survey.

Boyce et al. (2001) detected four known dIrr galaxies close to M 81. They also detected an HI cloud apparently devoid of stars, HIJASS J1021+6842. If anything else, this shows that blind HI surveys can still surprise and reveal objects which have been overlooked in one of the best studied and most surveyed nearby groups. Walter et al. (2005) present 
Table 1. Summary of targets for the VLA D-array observations

\begin{tabular}{|c|c|c|c|c|c|}
\hline \multirow[b]{2}{*}{ name } & \multirow[t]{2}{*}{ type } & RA $(2000.0)$ & \multirow{2}{*}{$\underset{\odot}{\mathrm{DEC}},(2000.0)$} & \multirow{2}{*}{$\begin{array}{l}\mathrm{m}_{\mathrm{B}} \\
\mathrm{mag}\end{array}$} & \multirow[b]{2}{*}{ detection } \\
\hline & & $\mathrm{h} \mathrm{m} \quad \mathrm{s}$ & & & \\
\hline KDG 61 & $\mathrm{sph}$ & 095703.1 & +683531 & 15.2 & $\mathrm{y}$ \\
\hline FM 1 & sph & 094510.0 & +684554 & 17.5 & $\mathrm{n}$ \\
\hline BK $5 \mathrm{~N}$ & sph & 100441.1 & +681522 & 17.4 & $\mathrm{y}$ \\
\hline KDG 64 & $\mathrm{sph}$ & 100701.9 & +674939 & 15.5 & $\mathrm{y}$ \\
\hline KK 77 & sph & 095010.5 & +673024 & 16.3 & $\mathrm{n}$ \\
\hline HIJASS & $?$ & 102100.0 & +684200 & $?$ & $\mathrm{y}$ \\
\hline DDO 71 & $\mathrm{sph}$ & 100506.4 & +663332 & 15.9 & $\mathrm{n}$ \\
\hline DDO 78 & sph & 102627.4 & +673916 & 15.8 & $\mathrm{n}$ \\
\hline BK $6 \mathrm{~N}$ & $\mathrm{sph}$ & 103429.8 & +660030 & 16.9 & $\mathrm{n}$ \\
\hline KKH 57 & sph & $\begin{array}{lll}10 & 00 & 15.9\end{array}$ & +631106 & 17.9 & $\mathrm{y}$ \\
\hline
\end{tabular}

VLA follow-up observations of HIJASS J1021+6842; Karachentsev \& Kaisin (2007) report the possible detection of an optical counterpart in the form of faint $\mathrm{H} \alpha$ emission which, if confirmed, would imply that although it would be one of the most extreme low surface brightness systems, HIJASS J1021+6842 wouldn't be a "Dark Galaxy".

In what follows we will report on a project which started out with the intention to study the HI content of previously catalogued dSph galaxies in the M 81 group. Deep observations were proposed to detect HI in these systems and outside the optical bodies in order to derive how much gas is associated with the galaxies themselves and how much is found in their immediate neighbourhood. We were interested in the latter to set constraints on the mechanisms that might be at work to remove gas from low-mass dwarfs. In the course of the project, however, we detected HI well beyond several of our targets, prompting us to change from targeted observations to blind survey mode.

\section{Observations and Results}

The observations reported here were made with the NRAO $†$ Very Large Array (VLA) over an extended period. Originally $9 \mathrm{dSph}$ galaxies, plus HIJASS J1021+6842 were observed in VLA D-array in February 2003 (see Table 1). In addition to mapping the source detected by Boyce et al. (2001), HI was detected in the fields of BK5N, KKH 57, KDG 61, and KDG 64. Follow-up C-array observations were obtained on these targets in April 2004. The resulting HI surface brightness maps of the combined C plus D-array observations are shown in Fig. 1. The maps were made using natural weighting for best sensitivity. They have an angular resolution of $35^{\prime \prime}$ which, at the assumed distance of the M 81 group of $3.63 \mathrm{Mpc}$ (Freedman et al. 2001), corresponds to $0.6 \mathrm{kpc}$. The rms noise is typically $0.6 \mathrm{mJy}_{\text {beam }}{ }^{-1}$ or $0.3 \mathrm{~K}$ at a velocity resolution of $5.2 \mathrm{~km} \mathrm{~s}^{-1}$. These single channel noise levels can be used to calculate a column density detection threshold. Assuming a signal to be genuine if it is detected at the $3 \sigma$ level across 3 channels, we find a minimum detectable column density of $2.5 \times 10^{19} \mathrm{~cm}^{-2}$. Using the same criterion we arrive at a minimum detectable $\mathrm{HI}$ mass for an $\mathrm{HI}$ cloud filling the beam, at the distance of the M 81 group, of $9 \times 10^{4} \mathrm{M}_{\odot}$.

The results of our observations can be summarised as follows:

KKH 57 We find a clear detection coinciding with the optical counterpart. The HI signal is only four channels wide at a systemic velocity of $203 \mathrm{~km} \mathrm{~s}^{-1}$; spatially the HI

$\dagger$ The National Radio Astronomy Observatory is a facility of the National Science Foundation operated under cooperative agreement by Associated Universities, Inc. 

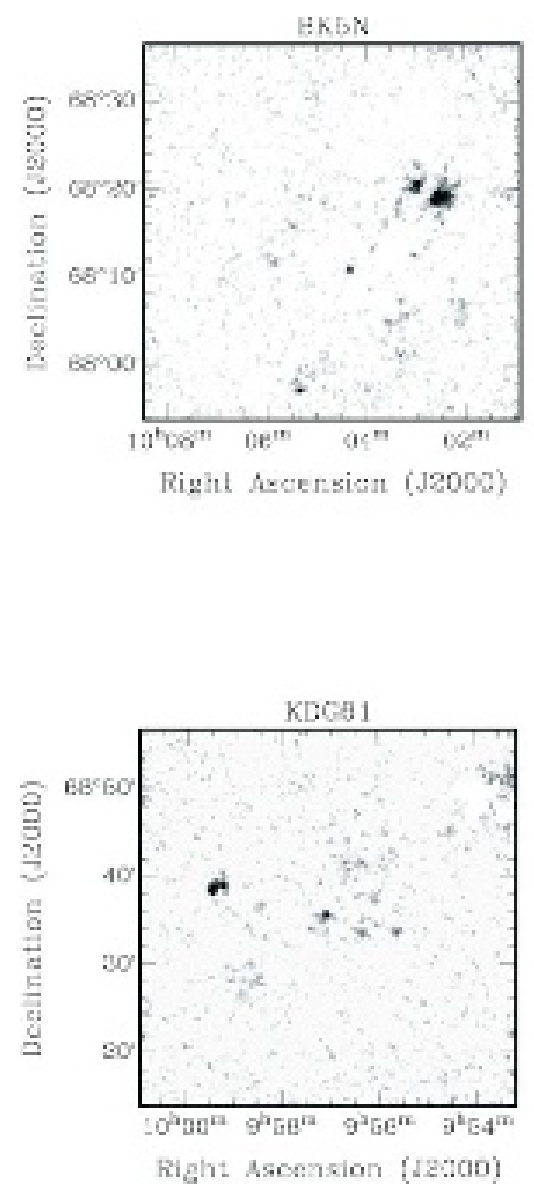
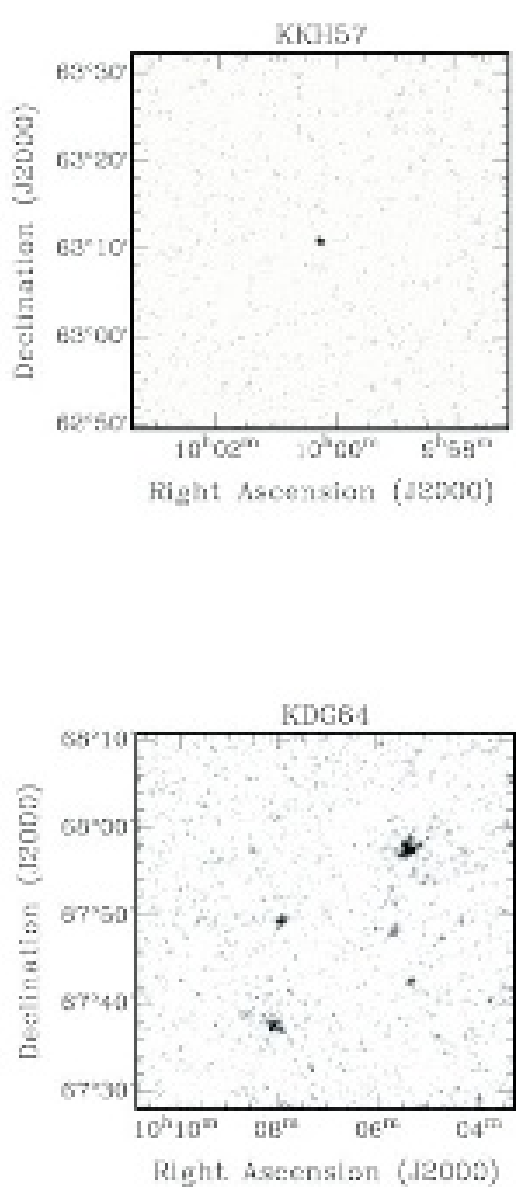

Figure 1. HI surface brightness maps of the four fields where HI emission was detected, BK5N, KKH 57, KDG 61, and KDG 64. KKH 57 is the only field where we detected HI associated with the target $\mathrm{dSph}$; in the other three fields, free-floating $\mathrm{HI}$ is found whereas the target galaxies remain undetected down to a level of $9 \times 10^{4} \mathrm{M}_{\odot}$ (based on a $3 \sigma$ detection threshold over 3 channels).

is unresolved. The low $\mathrm{M}_{\mathrm{HI}} / \mathrm{L}$ suggests this is a transition galaxy, i.e., an object which combines properties of a dSph, such as a low luminosity and prominent old stellar population, with those of a dIrr, e.g., the presence of HI (see Mateo 1998, for a more detailed treatise and Skillman et al. 2003, for examples). No other clouds are detected in this field.

KDG 61 Boyce et al. (2001) claim that this is a clear case of a transition galaxy. Their beam, however, is $12^{\prime}$ large and they are limited by confusion due to HI coming from the triplet. KDG 61 lies close to M 81 and, even with our much higher resolution data, it is not trivial to separate emission from KDG 61 or other field objects from M 81 emission. We seem to find several HI clouds which are neither related to the SE tidal arm nor to KDG 61 itself, at velocities ranging from -45 to $-15 \mathrm{~km} \mathrm{~s}^{-1}$.

BK5N Several HI clouds are detected with velocities from -130 to $-75 \mathrm{~km} \mathrm{~s}^{-1}$, well offset from the optical counterpart (for which no optical radial velocity has been published).

KDG 64 = UGC 5442 The optical galaxy lies at $-18 \pm 14 \mathrm{~km} \mathrm{~s}^{-1}$ (Simien \& Prugniel 2002). We find HI emission offset in position and velocity, with HI detected at velocities 


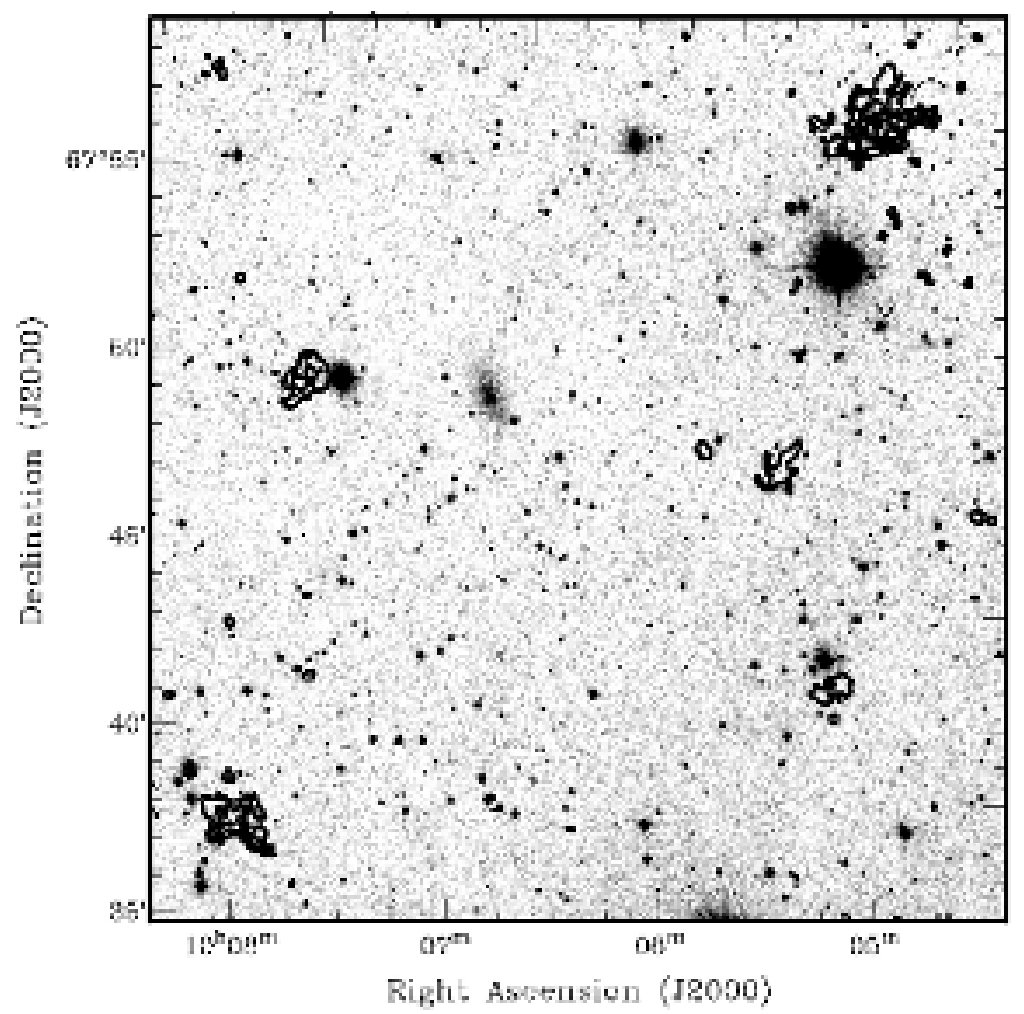

Figure 2. Optical image based on the Digitized Sky Survey (DSS) covering the field imaged in HI with the VLA centred on KDG 64. The contours indicate the locations of half a dozen free-floating HI clouds. Note that no HI is associated with KDG 64.

ranging from -120 to $-70 \mathrm{~km} \mathrm{~s}^{-1}$. This is illustrated in Fig. 2 which shows the location of the HI clouds with respect to the optical galaxy.

In summary, except for KKH57, none of the dSph targets were detected, confirming that most of them are devoid of HI. However quite surprisingly, HI was detected in several of the fields centred on a number of our targets but not obviously associated with them, neither in position nor in velocity. This then, of course, begs the question if these clouds are somehow associated with the dSph galaxies, or if they are semi-randomly distributed within the M 81 group. In order to investigate this, follow-up "blind" HI observations, of a further six fields were obtained with the VLA in D-configuration (observations taken in June, July, and August 2004). HI was found in only one of these fields, bordering BK5N and KDG 64 to the East.

In total, about a dozen barely resolved HI clouds were detected. They seem to predominantly occupy a region to the South-East of the triplet, extending as far away as $\sim 100 \mathrm{kpc}$. Their radial velocities are in the range -130 to $-70 \mathrm{~km} \mathrm{~s}^{-1}$. Velocity dispersions are $\sim 8 \mathrm{~km} \mathrm{~s}^{-1}$. HI masses are of order $10^{5} \mathrm{M}_{\odot}$. Figure 2 shows a DSS image of the field mapped around KDG64 with some of the HI clouds indicated with contours. No optical counterparts are visible on the DSS. The M 81 group has been surveyed several times in the optical down to levels considerably fainter than the DSS. In fact, it is one of the best studied nearby groups (e.g., Froebrich \& Meusinger, 2000). We can therefore be confident that these objects have very little starlight associated with them. 


\section{Discussion}

So what are they and what is their origin? There are several possibilities. They could be:

(a) primordial material which is infalling towards the M 81 triplet;

(b) High Velocity Cloud (HVC) analogues belonging to M 81;

(c) material originally part of BK5N and KDG 64 which has been expelled;

$(d)$ or tidal debris scattered into the SE quadrant.

Taking each of these possibilities in turn, the first explanation, infall of primordial material, is rather ad hoc. Without a determination of the metallicity of the gas in these HI clouds, it will be difficult to rule out this hypothesis. However, if primordial material were still to be around at column and space densities found here, it should have been detected in other nearby groups as well. No similar objects have been seen in deep HI observations, reaching comparable HI detection limits (Pisano et al. 2004, 2007).

If they represent analogues to HVCs, one would expect their velocity centroid to coincide with that of their host galaxy, M81. Also, their distribution would be expected to be more isotropic rather than that found here. No clouds were found in any of the other targeted observations nor in the blind pointings which together covered areas in and around the triplet. The third option, that they represent material that has been expelled from BK5N and KDG 64 is also hard to defend. Not only do the clouds seem uncorrelated spatially with both dwarfs, but in the one case where we have velocity information for the dwarf, the velocities of the clouds are offset substantially.

This leaves us with the last option, the clouds being tidal debris. Inspection of the HI observations by Appleton et al. (1981) shows that the HI clouds fall along the extension of their "feature VII", but beyond the area covered by either their survey or the VLA mosaic by Yun et al. (1994). Feature VII is prominent in the velocity range from -130 to $-100 \mathrm{~km} \mathrm{~s}^{-1}$, in broad agreement with the clouds found near BK5N and KDG 64. The VLA map by Yun et al. resolves feature VII, showing seemingly a spur branching off from the tidal bridge connecting NGC 3077 with M 81, which points roughly in the direction of the dwarf spheroidals. This is illustrated in Fig. 3 which shows Yun's HI surface brightness map as contours overlaid on an optical image. The numerical simulation published by Yun (1997) shows material from M 81 being dragged out by the passage of NGC 3077 and spread out towards the South-East. It therefore seems likely that the HI clouds found there are, in fact, tidal debris. They could be the neutral density peaks of a more dilute sheet of gas which is mostly ionised by the extragalactic radiation field, as a result of the column density being in general close to the value below which HI will become fully ionised. Peak column densities fall well below the canonical $\sim 10^{21} \mathrm{~cm}^{-2}$ threshold for star formation, explaining the lack of an optical counterpart.

\section{Conclusions}

In a search for $\mathrm{HI}$ in and around the lowest-mass dwarf spheroidal (dSph) galaxies in the M 81 group, we have discovered an unexpected population of HI clouds with masses of order $10^{5} \mathrm{M}_{\odot}$. Our observations of HIJASS J1021+6842 which were taken during the same observing runs were published separately (Walter et al. 2005). So far, about a dozen clouds have been detected within a region to the South-East of the triplet (in the vicinity of the dSphs KDG 64 and BK5N); there were no detections toward regions around other dSph galaxies in the same group and in five more "blind" pointings.

From a technical perspective, our observations go much deeper than any previous blind surveys done with single dish telescopes in the M 81 group (Lo \& Sargent 1979; 


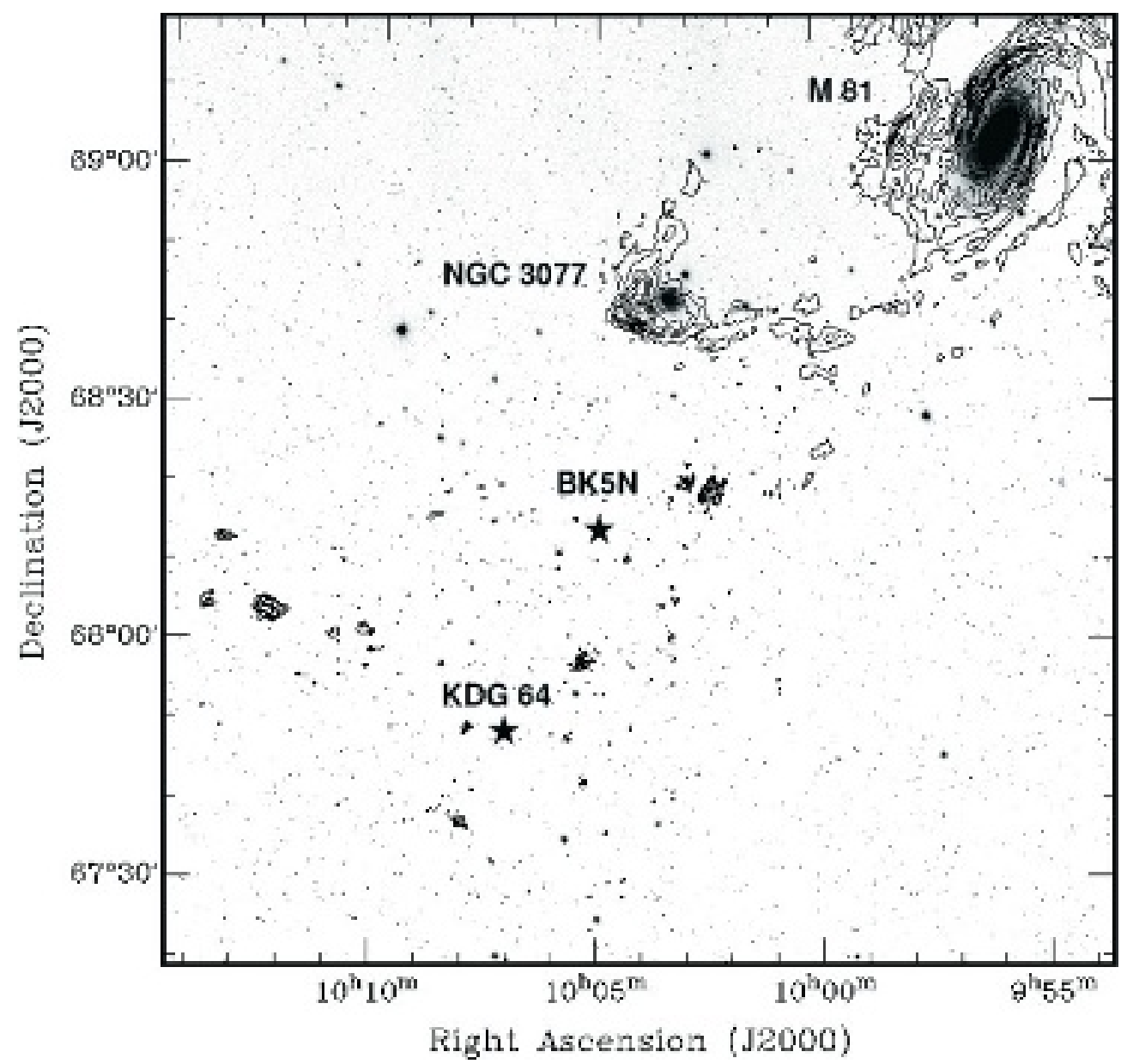

Figure 3. Optical image taken from the DSS of a $2^{\circ} \times 2^{\circ}$ field to the South-East of M 81 . Overlaid are contours of the HI distribution as measured by Yun et al. (1994), showing the tidal tail connecting M 81 with NGC 3077, and a "spur" pointing to the South-East. In addition we plot as contours the HI clouds detected in the VLA observations reported here that are seen in the direction of BK5N and KDG 64, plus the HI clouds encountered in VLA D-array observations in one of the "blind" pointings located due East of the two dwarf galaxies. The approximate locations of BK5N and KDG 64 are indicated with stars.

Boyce et al. 2001). The barely resolved clouds detected with the $35^{\prime \prime}$ VLA beam fall below the detection limit of single dish telescopes as a result of beam dilution. Our VLA data are also more sensitive by an order of magnitude than similar such surveys done in the Centaurus and Sculptor groups with the ATCA (de Blok et al. 2002; see also the discussion of the null result with Arecibo by Zwaan \& Briggs 2000).

The HI clouds detected here don't seem to have any optical counterparts. Circumstantial evidence argues in favour of these clouds being debris from the tidal interaction of the galaxies making up the M 81 triplet, notably the passage of NGC 3077 sweeping in a prograde fashion around the South of M 81. In order to better understand these enigmatic clouds further observations (extending the area around BK5N and KDG64 covered thus far) are being analysed. 


\section{Acknowledgements}

We thank Min Yun for providing us with an electronic version of his 1994 data which were used in Fig. 3.

\section{References}

Appleton, P. N., Davies, R. D. \& Stephenson, R. J. 1981, MNRAS 195, 327

Boyce, P. J., Minchin, R. F., Kilborn, V. A., Disney, M. J., Lang, R. H., Jordan, C. A., Grossi, M., Lyne, A. G., Cohen, R. J., Morison, I. M. \& Phillipps, S. 2001, ApJ (Letters) 560, L127

de Blok, W. J. G., Zwaan, M. A., Dijkstra, M., Briggs, F. H. \& Freeman, K. C. 2002, A\&A A 382, 43

Freedman, W. L., et al. 2001, ApJ 553, 47

Froebrich, D. \& Meusinger, H. 2000, A\&AS145, 229

Huchtmeier, W. K. \& Skillman, E. D. 1998, A\&AS 127, 269

Huchtmeier, W. K., Karachentsev, I. D., Karachentseva, V. E. \& Ehle, M. 2000, A\&\&AS 141, 469

Karachentsev, I. D. \& Kaisin, S. S. 2007, $A J 133,1883$

Lo, K. Y. \& Sargent, W. L. W. 1979, ApJ 227, 756

Mateo, M. L. 1998, ARAA 36, 435

Pisano, D. J., Barnes, D. G., Gibson, B. K., Staveley-Smith, L., Freeman, K. C. \& Kilborn, V. A. 2004 ApJ (Letter) 610, L17

Pisano, D. J., Barnes, D. G., Gibson, B. K., Staveley-Smith, L., Freeman, K. C. \& Kilborn, V. A. 2007 ApJ 662, 959

Simien, F. \& Prugniel, Ph. 2002, A\&\&A 384, 371

Skillman, E. D., Côté, S. \& Miller, B. W. 2003, AJ 125, 593

Yun, M. S., Ho, P. T. P. \& Lo, K. Y. 1994, Nature 372, 530

Yun, M. S. 1997, in: J. E. Barnes, and D. B. Sanders (eds.), Galaxy Interactions at Low and High Redshift Proceedings of IAU Symposium 186 p. 81

Yun, M. S., Ho, P. T. P. \& Lo, K. Y. 2000, in: J.G. Mangum \& S.J.E. Radford (eds.), Imaging at Radio through Submillimeter Wavelengths, ASP Conf. Proc. Vol. 217, p. 374

Walter, F., Skillman, E. D. \& Brinks, E. 2005, ApJ (Letters) 627, L105

Zwaan, M. A. \& Briggs, F. H. 2000, ApJ (Letters) 530, L61 